\title{
Efficacy of Gemcitabine and Bevacizumab Combination Chemotherapy for Platinum-Resistant Recurrent Ovarian Cancer: A Retrospective Analysis
}

\author{
Tatsuru Ohara ${ }^{1}$, Takamichi Sasaki ${ }^{1}$, Hiraku Endo ${ }^{1}$, Ryo Kanamori ${ }^{1}$, \\ Haruka Imai $^{1}$, Yuko Nagasawa ${ }^{1}$, Hiroyuki Yamanaka ${ }^{1}$, Shinji Hosonuma ${ }^{1}$, \\ Shiho Kuji ${ }^{1}$, Imari Deura ${ }^{1}$, Akiko Tozawa ${ }^{2}$, and Nao Suzuki ${ }^{1}$
}

(Received for Publication: April 14, 2021)

\begin{abstract}
Purpose: Bevacizumab and gemcitabine are key drugs for treating platinum-resistant recurrent ovarian cancer (PROC) patients. We retrospectively investigated its efficacy and safety of the combination chemotherapy in PROC patients.

Methods: Twenty-one patients who were diagnosed with PROC at our hospital that had received a course of multidrug therapy with gemcitabine and bevacizumab between March 2016-March 2018 were analyzed. Therapeutic effects were evaluated in accordance with the RECIST guidelines (version 1.1).

Results: Five patients had a complete response (CR), 5 had a partial response (PR), 8 had stable disease (SD), and 2 had progressive disease (PD). The response rate (CR + PR) was 50\%, and the disease-control rate was 90\%. The median progression-free survival (PFS) was 6 months (5-11 months), and the median overall survival (OS) was 11 months (11-23 months). Grade 3/4 hematological toxicities included neutropenia (Grade 3: 4 patients and Grade 4: 4 patients), anemia (Grade 3: 4 patients and Grade 4: 1 patient) and thrombocytopenia (Grade 3: 2 patients). No febrile neutropenia occurred. Grade 3 non-hematological toxicities included thromboembolism ( 2 patients), hypertension ( 2 patients), and interstitial pneumonia ( 1 patient). No Grade $\geq 3$ proteinuria or digestive tract perforation occurred.
\end{abstract}

Conclusions: The combination chemotherapy with gemcitabine and bevacizumab was feasible, effective and safe in PROC patients.

\section{Key words}

Ovarian cancer, bevacizumab, gemcitabine, recurrence, platinum-resistant

\section{Introduction}

Chemotherapy is the major treatment for relapsed epithelial ovarian cancer. It is known that the time from the end of the initial chemotherapy to recurrence (disease-free interval $[\mathrm{DFI}]$ ) is correlated with the response rate to chemotherapy in recurrent disease $^{1-3)}$.

Those who relapse within 6 months of the end of the initial chemotherapy, or who do not show any response to platinum-based chemotherapy, are classi- fied as having platinum-resistant ovarian cancer $(\mathrm{PROC})^{4,5)}$. Because it has been demonstrated that multidrug therapy including platinum preparations is superior to monodrug therapy, anticancer drugs without cross resistance are generally used for the initial chemotherapy, and monodrug therapy is mainly performed in consideration of drug toxicity ${ }^{6,7)}$.

In gynecological oncology, the antiangiogenic monoclonal antibody bevacizumab has improved therapeutic outcomes. In the AURELIA study on bevacizumab in platinum-resistant recurrent (PRR)

1 Department of Obstetrics and Gynecology, St. Marianna University School of Medicine

2 Department of Obstetrics and Gynecology, St. Marianna University Toyoko Hospital 
disease $^{8)}$, the primary endpoint, i.e., the median progression-free survival (PFS), was significantly extended to 6.7 month, and the hazard ratio (HR) was also significantly reduced to 0.48 . Multidrug therapy including bevacizumab is regarded as standard treatment for PROC.

It has been reported that monotherapy with gemcitabine is effective for PRR disease ${ }^{9,10)}$. However, there are only a limited number of reports on multidrug therapy with gemcitabine and bevacizumab (Bev-Gem therapy $)^{11,12)}$. Thus, we performed a retrospective study to evaluate the efficacy and safety of Bev-Gem therapy in PROC patients.

\section{Patients and Methods}

PROC patients treated with Bev-Gem therapy at our hospital between March 2016-March 2018 were identified by their medical records.

Gemcitabine was administered at a dose of 1000 $\mathrm{mg} / \mathrm{m}^{2}$ on days 1 and 8 , and bevacizumab at a dose of $15 \mathrm{mg} / \mathrm{m}^{2}$ on day 1 . Chemotherapy was scheduled to be repeated every 21 days. The eligibility criteria for subjects were as follows: neutrophil count $\geq 1500$ / $\mathrm{mm}^{3}$, platelet count $\geq 10.0 \times 10^{4} / \mathrm{mm}^{3}$, hemoglobin $\geq 9.0 \mathrm{~g} / \mathrm{L}$, non-hematological toxicity Grade $\leq 1$, and Eastern Cooperative Oncology Group Performance Status (ECOG PS) 0-2.

Treatment was delayed when granulocyte count was $<1500 / \mathrm{mm}^{3}$, the platelet count was $<10.0 \times$ $10^{4} / \mathrm{mm}^{3}$, or any non-hematological toxicity, excluding alopecia, malaise, nausea, and constipation, or grade 2 or higher occurred until the severity of symptoms was not higher than grade 1 .

Administration of bevacizumab was skipped when uncontrolled hypertension (systolic blood pressure, $>140 \mathrm{mmHg}$ or diastolic blood pressure, $>90$ $\mathrm{mmHg}$ ) or proteinuria (urine protein-to-creatinine ratio, $\geq 3.5$ ) was noted.

The gemcitabine dose was reduced to 800 $\mathrm{mg} / \mathrm{m} 2$ in response to febrile neutropenia, delay by 15-21 days because of prolonged toxicity, or grade 3 non-hematological toxicity. The bevacizumab dose was not reduced toxicity.

These treatments were continued for a maximum of six cycles, or until disease progression or the development of unacceptable toxicity.

Medical records were reviewed to investigate therapeutic effects and adverse events (AEs). Therapeutic effects were evaluated in accordance with the RECIST guidelines (version 1.1). The disease control rate (DCR) refers to the number of patients showing complete response (CR), partial response (PR) or stable disease (SD) /total number of patients. Adverse events were assessed in accordance with the National Cancer Institute Common Terminology Criteria for Adverse events (NCI-CTCAE, version 4.0). Tumors were evaluated using contrast-enhanced computed tomography every $2-3$ cycles.

Progression-free survival (PFS) refers to the time from the start of Bev-Gem therapy to lesion progression or death. The overall survival (OS) refers to the time from the start of Bev-Gem therapy to death.

PFS was evaluated as a primary endpoint, and response rate, clinical benefit rate, adverse events, OS were evaluated as secondary endpoints. Prior to this study, the number of patients treated with bevacizumab (bevacizumab beyond first progression [BBP] cases) was examined, and the PFS and OS were compared with the group treated without bevacizumab (non-BBP cases).

For statistical analyses, JMP ${ }^{\mathbb{R}}$ Pro (SAS Institute Inc., NC, USA) was used. The survival rate and disease-free survival rate were calculated using the $\mathrm{Ka}$ plan-Meier method and were statistically analyzed using the long-rank test.

This study was approved by the Ethics Committee of St. Marianna University School of Medicine (approval number: 3735).

\section{Results}

Overall, 21 patients were enrolled. The characteristics of the are shown in Table 1. The median observation time was 11 months (4-26 months). The median age was 70 y (47-75 y). The performance status (PS) was Level 0 in 18 patients $(86 \%)$ and Level 1 in 3 patients (14\%). There were 15 ovarian cancer patients (4 at Stage I, 2 at Stage II, 7 at Stage III and 2 at Stage IV) and 6 peritoneal cancer cases patients ( 3 at Stage III and 3 at Stage IV). There were 14 high-grade serous carcinoma patients, 5 clear cell cancer patients, and 2 endometrioid carcinoma patients. The platinum-free interval (PFI) was $<3$ months in 11 patients and 3-6 months in 10 patients.

The number of previous treatment regimens was 1 and 2 in 19 patients (91\%), 3 in 1 patient (5\%), and 4 in 1 patient (5\%). The median number of bevacizumab doses was 6 (1-13). There were 7 patients who had been treated with bevacizumab prior to this study (BBP cases). Ten patients (47.6\%) needed to skip treatments, and 2 patients $(9.5 \%)$ needed gemcitabine dose reduction.

In 20 patients with evaluable lesion (1 patient 
Efficacy of gemcitabine and bevacizumab combination chemotherapy for platinum-resistant recurrent ovarian cancer: a retrospective analysis

Table 1. Characteristics of Patients Treated with the Combination of Bevacizumab and Gemcitabine

\begin{tabular}{|c|c|c|}
\hline \multirow[t]{2}{*}{ Characteristics } & \multicolumn{2}{|c|}{ Bevacizumab and gemcitabine $(n=21)$} \\
\hline & Number & $\%$ \\
\hline \multicolumn{3}{|l|}{ Age } \\
\hline Median, y (range) & $70(47-75)$ & \\
\hline \multicolumn{3}{|l|}{ Performance status } \\
\hline 0 & 18 & 86 \\
\hline 1 & 3 & 14 \\
\hline 2 & 0 & \\
\hline 3 & 0 & \\
\hline \multicolumn{3}{|l|}{ Primary site } \\
\hline Ovary & 15 & 71 \\
\hline Peritoneum & 6 & 29 \\
\hline \multicolumn{3}{|l|}{ FIGO stage } \\
\hline I & 4 & 19 \\
\hline II & 2 & 10 \\
\hline III & 10 & 47 \\
\hline IV & 5 & 24 \\
\hline \multicolumn{3}{|l|}{ Histology } \\
\hline Serous carcinoma & 14 & 66 \\
\hline Clear cell carcinoma & 5 & 24 \\
\hline Endometrioid carcinoma & 2 & 10 \\
\hline \multicolumn{3}{|l|}{ Platinum-free interval } \\
\hline$<3$ months & 11 & 52 \\
\hline 3 months -6 months & 10 & 48 \\
\hline \multicolumn{3}{|l|}{ Prior chemotherapy regimens } \\
\hline 1 & 10 & 48 \\
\hline 2 & 9 & 42 \\
\hline 3 & 1 & 5 \\
\hline 4 & 1 & 5 \\
\hline $\begin{array}{l}\text { Previous chemotherapy with bevacizumab } \\
\qquad \text { (BBP *cases) }\end{array}$ & 7 & 33 \\
\hline \multicolumn{3}{|l|}{ No. of cycles } \\
\hline median (range) & $6(1-13)$ & \\
\hline Skipped & 10 & 47.6 \\
\hline Dose modification & 2 & 9.5 \\
\hline
\end{tabular}

* bevacizumab beyond first progression

had no evaluable lesion), there were 5 of CR, 5 of $\mathrm{PR}, 8$ of SD, 2 of progressive disease (PD). The response rate was $50 \%$ and the clinical benefit rate was 90\% (Table 2).

Grade 3/4 hematological toxicity was as follows: neutropenia was seen in 8 patients $(38.1 \%)$, with anemia in 5 (23.8\%), and thrombocytopenia in 2 $(9.5 \%)$. No febrile neutropenia occurred (Table 3 ).
Grade 3 thromboembolism was seen in 2 patients (9.5\%), with Grade 3 hypertension in 3 (14.2\%) and Grade 3 interstitial pneumonia in 1 $(4.8 \%)$. Also, Grades 1 and 2 proteinuria were seen in $7(33.3 \%)$ and $4(19.0 \%)$, respectively. No Grade 3 proteinuria occurred.

Grade 2 seizure activity was seen 1 patient (4.8\%). No digestive tract perforation, a common $\mathrm{AE}$ 
Table 2. Response to Bevacizumab and Gemcitabine

\begin{tabular}{lc}
\hline & Bevacizumab and gemcitabine evaluable cases $(\mathrm{n}=20)$ \\
\hline Response & 5 \\
Complete response & 5 \\
Partial response & 8 \\
Stable disease & 2 \\
Progressive disease & $50 \%$ \\
Response rate & $90 \%$ \\
Disease-control rate & \\
\hline
\end{tabular}

Table 3. Adverse Effects in Patients Treated with Combined Bevacizumab and Gemcitabine

\begin{tabular}{lccccc}
\hline & \multicolumn{5}{c}{ Bevacizumab and gemcitabine $(\mathrm{n}=21)$} \\
\cline { 2 - 5 } & $\mathrm{G} 1$ & $\mathrm{G} 2$ & $\mathrm{G} 3$ & $\mathrm{G} 4$ & Grade $\geq 3(\%)$ \\
\hline Neutropenia & 4 & 2 & 4 & 4 & 38 \\
Leukopenia & 4 & 3 & 2 & 1 & 14 \\
Anemia & 4 & 2 & 4 & 1 & 24 \\
Thrombocytopenia & 7 & 1 & 2 & 0 & 10 \\
Febrile neutropenia & - & - & 0 & 0 & 0 \\
Hypertension & 2 & 0 & 3 & 0 & 14 \\
Proteinuria & 7 & 4 & 0 & 0 & 9 \\
Bleeding & 0 & 0 & 0 & 0 & 0 \\
Thromboembolic event & 0 & 0 & 2 & 0 & 10 \\
Seizure & 0 & 1 & 0 & 0 & 0 \\
Pneumonia & 0 & 0 & 1 & 0 & 5 \\
Colonic obstruction & 0 & 0 & 1 & 0 & 5 \\
Gastrointestinal perforation & 0 & 0 & 0 & 0 & 0 \\
Fistula & 0 & 0 & 0 & 0 & 0 \\
\hline & & & & 0 & 5 \\
\hline
\end{tabular}

in bevacizumab therapy, occurred (Table 3).

Bev-Gem therapy was discontinued in 4 patients (19\%) for the following reasons other than the progression of existing lesions: interstitial pneumonia in 1 ; seizure activity in 1 ; cerebral infarction in 1 ; and deep vein thrombosis in 1 .

The median PFS was 6 months (Figure 1A), and the median OS was 15 months (Figure 1B).

The median PFS of BBP cases was 5 months (Figure 2A), and median OS was 11 months (Figure 2B). The median PFS of non-BBP cases was 9 months (Figure 2A), and median OS was 22 months (Figure 2B).

\section{Discussion}

In the AURELIA study, multidrug therapy with bevacizumab and other anticancer drugs improved the PFS of platinum-sensitive recurrent ovarian cancer (PSR) patients but did not improve the $\mathrm{OS}^{8)}$. However, the results of subanalyses suggested that only multidrug therapy with bevacizumab and paclitaxel improved both PFS and $\mathrm{OS}^{13)}$. Nagao et al. reported that when Ben-Gem therapy was administered to PSR patients, the median PFS and OS were 5.1 months and 21.3 months, respectively, and that BenGem therapy was effective and safe ${ }^{12)}$.

The median PFS and OS in the present study 

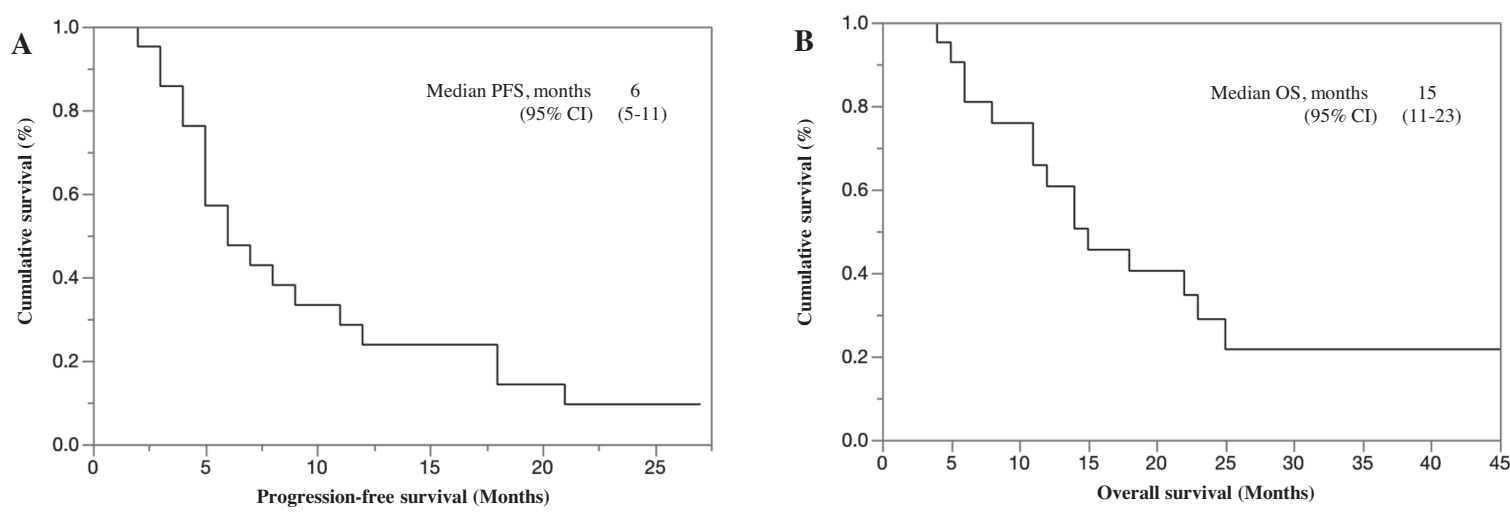

Figure 1. Kaplan-Meier curves for Progression-free survival (PFS) and overall survival (OS) in patients receiving bevacizumab and gemcitabine (Bev-Gem). PFS (A) and OS (B)
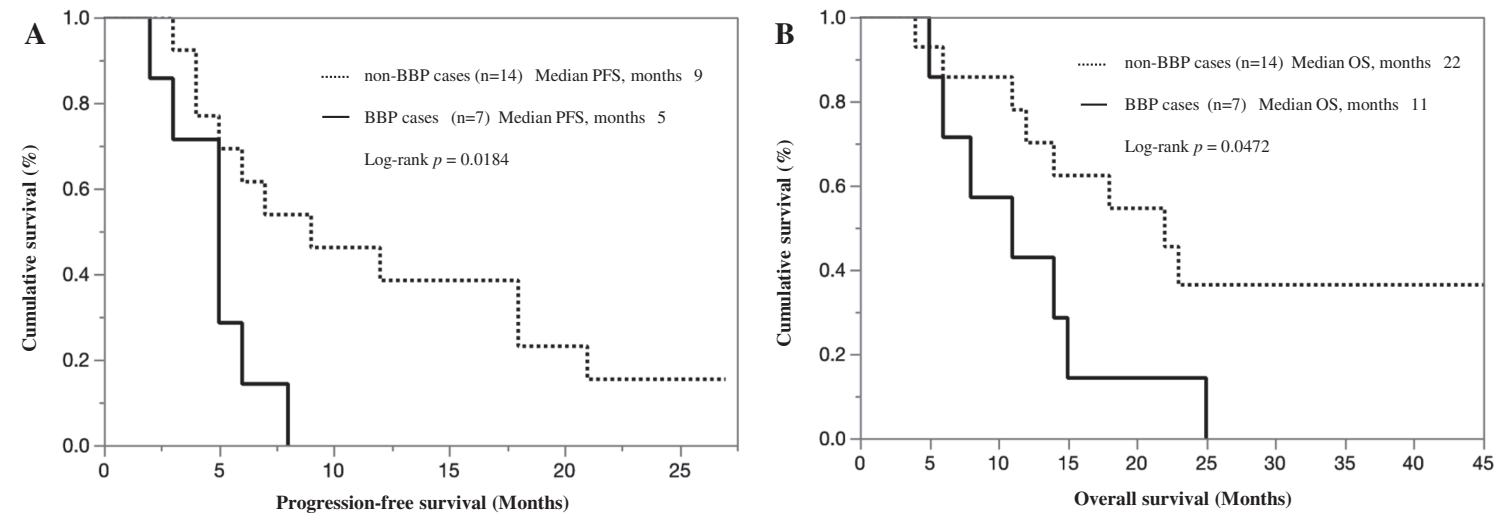

Figure 2. Kaplan-Meier curves for Progression-free survival (PFS) and overall survival (OS) in bevacizumab beyond first progression (BBP) cases and non-BBP cases. PFS (A) and OS (B).

were 6 months and 15 months, respectively, and were comparable to those in the AURELIA study. In 2016 we reported on a gemcitabine monotherapy to assess the efficacy and safety in PROC patients. The median PFS and OS were 3.5 months and 8 months ${ }^{14)}$. This suggests that Bev-Gem therapy may become the preferred multidrug therapy for PRR disease.

Bevacizumab is associated with gastrointestinal perforation, thromboembolism, fistula, delayed wound healing, and tumor-associated bleeding. When bevacizumab is used for ovarian cancer treatment, sufficient attention should be paid to the risk of gastrointestinal perforation. It has been reported that the risk factors for gastrointestinal perforation include surgery, inflammatory bowel disease, and administration of $\mathrm{Bev}^{15}$.

In this study, although Grade 3/4 neutropenia was seen in 8 patients (38.1\%), no febrile neutropenia occurred. This suggests that Bev-Gem therapy is highly tolerable. Although Grade 3 hypertension was seen in 3 patients (14.2\%), Bev-Gem therapy could be continued in combination with antihypertensive drugs for blood pressure control. Grade 3 thromboembolism was seen in 2 patients $(9.5 \%)$; one developed cerebral infarction (Trousseau's syndrome) associated with aggravation of tumors during Cycle 1 and withdrew from Bev-Gem therapy, and the other developed deep vein thrombosis (DVT) of the right lower limb during Cycle 4 and withdrew from BevGem therapy because the oral anticoagulant drug the patient was taking made it difficult to control thromboembolism. Grade 3 interstitial pneumonia was seen in 1 patient (4.8\%) after Cycle 6 and was felt to be related to gemcitabine. The Grade 3 interstitial pneumonia spontaneously resolved after withdrawal from gemcitabine, and with a change in the regimen, treatment could be continued. Grade 2 seizure activity was seen in 1 patient (4.8\%) during Cycle 4, with tet- 
any lasting approximately 5 minutes. A head computed tomography (CT) and electroencephalogram were negative. Bev-Gem therapy was discontinued at the judgment of the physician in charge.

Because vascular endothelial growth factor (VEGF) is an important mediator in the course of tumor progression, it is important to continuously inhibit VEGF after tumor progression. This confirms Mancuso et al.'s finding that maintenance treatment with bevacizumab (BBP) has a role in cancer therapy ${ }^{16)}$.

The BRiTE study ${ }^{17)}$ and ML18147 study ${ }^{18)}$ were conducted in bowel cancer patients, and the TANIA study ${ }^{19)}$ in breast cancer patients. The results of these studies suggested that BBP is clinically useful in these malignancies.

In BBP for ovarian cancer, the results of the MITO16 study/MANGO2b study ${ }^{20)}$ were published at an ASCO meeting in 2018. These studies were conducted in patients that developed relapsed PSR $\geq 6$ months after the end of the first line chemotherapy/ bevacizumab therapy. The median PFS (primary endpoint) was significantly extended in the chemotherapy/bevacizumab therapy group compared to the chemotherapy group (8.8 months vs. 11.8 months, HR $0.51,95 \%$ CI $0.41-0.65, \log$-rank $\mathrm{P}<.001)$, indicating the efficacy of BBP in PSR. It is problematic that PRR is not avoidable in the course of ovarian cancer treatment. The results of the JGOG3023 study on BBP in PRR are awaited ${ }^{21}$.

Although BBP was seen in 7 patients (33\%), the PFS and OS do not suggest that BBP is effective (Figure 2). The PFI was $<3$ months in 4 patients. Because the 4 patients were resistant to chemotherapy, BBP was not considered to be effective. The JGOG3023 study on the usefulness of BBP in PRR is being conducted in Japan, and the results of the study are also awaited ${ }^{20)}$.

\section{Conclusion}

Bev-GEM therapy improved PFS in the PROC patients. AEs of Bev-Gem therapy was tolerable. BEV-Gem therapy could be a choice of new treatment for PROC.

\section{Conflicts of Interest}

The authors have nothing to disclose.

\section{References}

1) Colombo N, Gore M. Treatment of recurrent ovarian cancer relapsing 6-12 months post plati- num-based chemotherapy. Crit Rev Oncol Hematol 2007; 64: 129-138.

2) Markman M, Markman J, Webster K, et al. Duration of response to second-line, platinumbased chemotherapy for ovarian cancer: implications for patient management and clinical trial design. J Clin Oncol 2004; 22: 3120-3125.

3) Pfisterer J, Vergote I, Du Bois A, et al. Combination therapy with gemcitabine and carboplatin in recurrent ovarian cancer. Int $\mathbf{J}$ Gynecol Cancer 2005; 15: 36-41.

4) Markman M, Rothman R, Hakes T, et al. Second-line platinum therapy in patients with ovarian cancer previously treated with cisplatin. J Clin Oncol 1991; 9: 389-393.

5) Harries M, Gore M. Part II: Chemotherapy for epithelial ovarian cancer-treatment of recurrent disease. Lancet Oncol 2002; 3: 537-545.

6) Buda A, Floriani I, Rossi R, et al. Randomised controlled trial comparing single agent paclitaxel vs epidoxorubicin plus paclitaxel in patients with advanced ovarian cancer in early progression after platinum-based chemotherapy: an Italian Collaborative Study from the Mario Negri Institute, Milan, G.O.N.O (Gruppo Oncologico Nord Ovest) group and I.O.R. (Istituto Oncologico Romagnolo) group. Br J Cancer 2004; 90: 2112-2117.

7) Sehouli J, Stengel D, Oskay-Oezcelik G, et al. Nonplatinum topotecan combinations versus topotecan alone for recurrent ovarian cancer: results of phase III study of the North-Eastern German Society Gynecological Oncology Ovarian Cancer Study Group. J Clin Oncol 2008; 26: 3176-3182.

8) Pujade-Lauraine E, Hilpert F, Weber B, et al. Bevacizumab combined with chemotherapy for platinum-resistant recurrent ovarian cancer: The AURELIA open-label randomized phase III trial. J Clin Oncol 2014; 32: 1302-1308.

9) Mutch DG, Orlando M, Goss T, et al. Randomised Phase III trial of gemcitabine compared with pegylated liposomal doxorubicin in patients with platinum-resistant ovarian cancer. J Clin Oncol 2007; 25: 2811-2818.

10) Ferrandina N, Ludovisi M, Lorusso D, et al. Phase III trial of gemcitabine compared with pegylated liposomal doxorubicin in progressive or recurrent ovarian cancer. J Clin Oncol 2008; 26: 890-896.

11) Takasaki K, Miyamoto M, Takano M, et al. Ad- 
dition of bevacizumab to gemcitabine for platinum-resistant recurrent ovarian cancer: a retrospective analysis. Cancer Chemother Pharmacol 2018; 81: 809-814.

12) Nagao S, Kogiku A, Suzuki K, et al. A phase II study of the combination chemotherapy of bevacizumab and gemcitabine in women with platinum-resistant recurrent epithelial ovarian, primary peritoneal, or fallopian tube cancer. J Ovarian Res 2020; 13: 14.

13) Poveda AM, Selle F, Hilpert F, et all. Bevacizumab combined with weekly paclitaxel, pegylated liposomal doxorubicin, or topotecan in platinum-resistant recurrent ovarian cancer: analysis by chemotherapy cohort of the randomized phase III AURELIA trial. J Clin Oncol 2015; 33: 3836-3838.

14) Kondo A, Hatano M, Miura A, et al. Study of efficacy and safety of gemcitabine for platinumresistant recurrent ovarian cancer in our hospital. Kanagawa Journal of Obstetrics and Gynecology 2016; 53: 70-73.

15) Burger RA, Brady MF, Bookman MA, et al. Risk factors for GI adverse events in a phase III randomized trial of bevacizumab in first-line therapy of advanced ovarian cancer: A Gynecologic Oncology Group Study. J Clin Oncol 2014; 20: 1210-1217.

16) Mancuso MR, Davis R, Norberg SM, et al. Rapid vascular regrowth in tumors after reversal of VEGF inhibition. J Clin Invest 2006; 116: 2610-2621.

17) Grothey A, Sugrue MM, Purdie DM, et al. Bevacizumab beyond first progression is associated with prolonged overall survival in metastatic colorectal cancer: results from a large observational cohort study (BRiTE). J Clin Oncol 2008; 20: 5326-5334.

18) Bennouna J, Sastre J, Arnold D, et al. Continuation of bevacizumab after first progression in metastatic colorectal cancer (ML18147): a randomised phase 3 trial. Lancet Oncol 2013; 14: 29-37.

19) von Minckwitz G, Puglisi F, Cortes J, et al. Bevacizumab plus chemotherapy versus chemotherapy alone as second-line treatment for patients with HER2-negative locally recurrent or metastatic breast cancer after first-line treatment with bevacizumab plus chemotherapy (TANIA): an open-label, randomised phase 3 trial. Lancet Oncol 2014; 15: 1269-1278.

20) Pignata S, Lorusso D, Joly F, et al. Chemotherapy plus or minus bevacizumab for platinumsensitive ovarian cancer patients recurring after a bevacizumab containing first line treatment: The randomized phase 3 trial MITO16BMaNGO OV2B-ENGOT OV17. J Clin Oncol 2018; 36 (15_suppl): 5506. doi: 10.1200/JCO. 2018.36.15_suppl.5506.

21) Shoji T, Komiyama S, Kigawa J, et al. An openlabel, randomized, phase II trial evaluating the efficacy and safety of standard of care with or without bevacizumab in platinum-resistant epithelial ovarian, fallopian tube, or primary peritoneal cancer patients previously treated with bevacizumab for front-line or platinum-sensitive ovarian cancer: rationale, design, and methods of the Japanese gynecologic oncology group study JGOG3023. BMC Cancer 2018; 18: 771. 